\title{
Learning curves of theta/beta neurofeedback in children with ADHD
}

\author{
Tieme W. P. Janssen ${ }^{1} \cdot$ Marleen Bink $^{1} \cdot$ Wouter D. Weeda ${ }^{2} \cdot$ Katleen Geladé $^{1} \cdot$ \\ Rosa van Mourik ${ }^{4} \cdot$ Athanasios Maras $^{3} \cdot$ Jaap Oosterlaan $^{1}$
}

Received: 7 June 2016 / Accepted: 15 November 2016 / Published online: 19 November 2016

(C) The Author(s) 2016. This article is published with open access at Springerlink.com

\begin{abstract}
Neurofeedback is widely applied as non-pharmacological intervention aimed at reducing symptoms of ADHD, even though efficacy has not been unequivocally established. Neuronal changes during the neurofeedback intervention that resemble learning can provide crucial evidence for the feasibility and specificity of this intervention. A total of 38 children (aged between 7 and 13 years) with a DSM-IV-TR diagnosis of ADHD, completed on average 29 sessions of theta $(4-8 \mathrm{~Hz}) /$ beta $(13-20 \mathrm{~Hz})$ neurofeedback training. Dependent variables included training-related measures as well as theta and beta power during baseline and training runs for each session. Learning effects were analyzed both within and between sessions. To further specify findings, individual learning curves were explored and correlated with behavioral changes in ADHD symptoms. Over the course of the training, there was a linear increase in participants' mean
\end{abstract}

M. Bink as a shared first authorship.

Electronic supplementary material The online version of this article (doi:10.1007/s00787-016-0920-8) contains supplementary material, which is available to authorized users.

Tieme W. P. Janssen

twp.janssen@vu.nl

1 Clinical Neuropsychology Section, Vrije Universiteit, Van Der Boechorststraat 1, 1081 BT Amsterdam, The Netherlands

2 Department of Psychology, Leiden University, Wassenaarseweg 52, 2333 AK Leiden, The Netherlands

3 Yulius Academy, Dennenhout 1, 2994 GC Barendracht, The Netherlands

4 Royal Dutch Kentalis, Vlampijpstraat 78, 3534 AR Utrecht, The Netherlands training level, highest obtained training level and the number of earned credits (range $b=0.059,-0.750, p<0.001$ ). Theta remained unchanged over the course of the training, while beta activity increased linearly within training sessions $(b=0.004,95 \% \mathrm{CI}=[0.0013-0.0067], p=0.005)$ and over the course of the intervention $(b=0.0052,95 \%$ $\mathrm{CI}=[0.0039-0.0065], p<0.001)$. In contrast to the group analyses, significant individual learning curves were found for both theta and beta over the course of the intervention in 39 and 53\%, respectively. Individual learning curves were not significantly correlated with behavioral changes. This study shows that children with ADHD can gain control over EEG states during neurofeedback, although a lack of behavioral correlates may indicate insufficient transfer to daily functioning, or to confounding reinforcement of electromyographic activity. Clinical Trials Registration: This trial is registered at the US National Institutes of Health (ClinicalTrials.gov, ref. no: NCT01363544); https://clinicaltrials.gov/show/NCT01363544.

Keywords Neurofeedback - Theta/beta-training - ADHD . Paediatric $\cdot$ EEG $\cdot$ Learning curves

\section{Introduction}

Attention deficit hyperactivity disorder (ADHD) is a neuropsychiatric disorder that is characterized by a persistent pattern of inattentive and/or hyperactive and impulsive behavior that interferes with normal social, academic or occupational functioning [1, 2]. Currently, the most commonly applied intervention for ADHD is treatment with stimulant medication. Although stimulant medication is effective in short-term symptom reduction [3, 4], there is limited knowledge on long-term effectiveness and possible 
long-term negative effects [5]. Therefore, parents may be reluctant to agree with stimulant treatment for their child [6]. This situation urges the need for effective alternatives to pharmacological interventions. However, few alternative interventions prove efficacious when only probably blinded results are considered [7], which might indicate a considerable contribution of non-specific effects to the positive outcomes reported for alternative interventions. Research into working mechanisms of alternative interventions may help to distinguish effective from non-effective treatment elements, which may contribute to improving and developing nonpharmacological interventions for children with ADHD.

Neurofeedback is considered a promising non-pharmacological alternative for the treatment of ADHD. The most commonly used type of neurofeedback is based on electroencephalogram (EEG) activity of the patient and is, therefore, also called EEG biofeedback. During neurofeedback training, patients receive feedback of their current brain activity. Using operant conditioning principles, the aim is that patients learn to modify the activation state of their brain. Children with ADHD may have atypical EEG power spectra, showing increased theta and decreased beta activity $[8,9]$. On a behavioral level, theta has been negatively related to alertness, whereas beta has been positively related to attention [10-12]. Accordingly, one of the most commonly applied neurofeedback protocol aims to decrease theta (4-8 Hz) activity and increase beta (13$20 \mathrm{~Hz}$ ) activity [12-15]. Behavioral outcomes for theta/beta neurofeedback in children with ADHD vary across studies. Whereas unblinded randomized controlled trials (RCT) reveal superiority of neurofeedback over control conditions $[16,17]$, blinded placebo-controlled studies show similar improvements in behavior for neurofeedback and shamneurofeedback [18-20].

Surprisingly, data on training progress within and between neurofeedback sessions are scarcely considered in ADHD [21] and the available results are mixed. Uncontrolled studies showed that a majority of children with ADHD who displayed elevated theta/beta ratios before the training were able to decrease theta [22] or theta/beta ratio over the course of theta/beta neurofeedback training [23]. In contrast, another study showed that children with ADHD were not able to decrease theta/beta ratios over the training sessions [24]. Only two RCT studies reported on training data of theta/beta protocols. The first study of Vollebregt, van Dongen-Boomsma, Buitelaar, and SlaatsWillemse [20] analyzed a small subsample of ten children who received different forms of theta/beta neurofeedback. Seven out of ten children showed the desired change over time in either theta or beta power. However, all ten children showed also changes in one frequency band in the opposite of the trained direction. The second study [25] showed that adolescents who received theta/sensorimotor rhythm neurofeedback (SMR; 12-15 Hz) became better in suppressing theta within training sessions. However, the mean value of theta did not decrease over the entire treatment. Both studies [20, 25] reported behavioral improvements over time, irrespective of whether the children received an active form of theta/beta neurofeedback training or not. In conclusion, the current literature remains ambiguous to what extend theta and beta activity can be trained in children with ADHD and whether such training effects underlie the behavioral changes observed.

Other important aspects of neurofeedback concern stability and generalizability of trained frequencies. If children with ADHD are able to adapt theta or beta activity, the question is whether this leads to sustainable changes that also generalize to situations outside neurofeedback training sessions. Two RCT studies indeed reported a linear decrease in theta activity at midline scalp electrodes after the training was completed [26, 27]. In contrast, another study did not find any differences in theta or beta activity for children following individualized theta/beta neurofeedback [28]. Alternatively, when it seems that neurofeedback results mainly in state changes, it is of clinical importance to know whether this temporary state can be generalized to daily activities such as school activities. To promote generalizability to daily life, some neurofeedback training programs apply transfer trials. During these trials, patients receive feedback on their performance after the trial is completed and do not receive feedback during the trial. Additionally, transfer cards, with a visual representation of the neurofeedback training screen, may be given to patients to use in daily life. Transfer cards are supposed to evoke the desired decreased theta/beta ratio as a conditioned response and may thus be used to improve attention, for example during school assignments.

In summary, several studies investigated the behavioral and neurocognitive outcomes of theta/beta neurofeedback; however, little is known about specific working mechanisms of theta/beta neurofeedback. Therefore, the aim of the current study was to explore the effects of theta/beta neurofeedback on EEG activity within and between neurofeedback sessions over the course of a 10-week, 30 sessions theta/beta neurofeedback intervention in children with ADHD. More specifically, both training parameters between sessions (mean and maximum training level), and theta and beta power within and between training sessions, were tested for learning effects. Beside these group analyses, we identified individual learners as participants that were able to significantly change theta or beta power in the desired direction. Generalizability was explored by comparing transfer and non-transfer trials, and baseline versus training EEG. Additionally, we explored whether the ability to adapt EEG theta or beta activity was related to ADHD symptom reductions. 
Table 1 Group characteristics

\begin{tabular}{lllll}
\hline & $N$ & Mean & SD & Range \\
\hline Age & 0038 & 9.87 & 1.81 & 7.2 to 13.6 \\
Gender (male/female) & 0038 & $29 / 9$ & & \\
IQ & 0038 & 100.45 & 13.34 & 81 to 134 \\
DBDRS parent & & & & \\
$\quad$ Inattention & 0038 & 16.63 & 5.15 & 8 to 26 \\
$\quad$ Hyperactivity/impulsivity & 0038 & 14.50 & 5.99 & 3 to 25 \\
DBDRS teacher & & & & \\
$\quad$ Inattention & 0038 & 15.37 & 5.29 & 4 to 25 \\
$\quad$ Hyperactivity/impulsivity & 0038 & 13.79 & 6.90 & 3 to 26 \\
SWAN parent & & & & \\
$\quad$ Inattention & 0038 & 1.44 & 0.51 & 0.22 to 2.33 \\
$\quad$ Hyperactivity/impulsivity & 0038 & 1.30 & 0.71 & -0.89 to 2.33 \\
SWAN teacher & & & & \\
$\quad$ Inattention & 0037 & 1.37 & 0.91 & -0.67 to 2.89 \\
$\quad$ Hyperactivity/impulsivity & 0037 & 1.15 & 0.92 & -1.11 to 2.78 \\
\hline
\end{tabular}

DBDRS disruptive behavior disorders rating scale (raw scores), strengths and weaknesses of ADHD symptoms and normal behavior scale (SWAN), SD standard deviation

\section{Materials and methods}

\section{Participants}

This study pertains to a subsample of 38 children that were randomized to neurofeedback intervention in a multicenter three-way parallel group study with balanced randomization into the effects of neurofeedback compared to optimally titrated methylphenidate and physical activity (applied as a semi-active control condition) in children with ADHD (Clinical Trials Registration: ClinicalTrials. gov, Identifier: NCT01363544). Of the 38 children, 7 had comorbid disorders including learning disorders $(n=3)$, autism spectrum disorders (ASS; $n=2$ ), learning disorder combined with ASS $(n=1)$, and learning disorder combined with anxiety disorder $(n=1)$. Group characteristics are listed in Table 1. For further details, please see Janssen et al. [27].

\section{Neurofeedback intervention}

Neurofeedback consisted of three individual training sessions a week, with each session lasting $45 \mathrm{~min}$ including $20 \mathrm{~min}$ of effective training, over a period of 10 to 12 weeks. The mean number of completed training sessions was $29(M=28.53, \mathrm{SD}=2.63$, range 19-30). Each training session consisted of 10 runs and started with a 1-min baseline theta/beta index measurement. This baseline measurement was used in the following 10 runs of neurofeedback. In sum, theta and beta were recorded during these runs and children were rewarded with credits related to the size of the improvements compared to baseline. See Fig. 1 for a schematic representation of the neurofeedback intervention.

In more detail, theta/beta training was applied unidirectionally, with the aim to inhibit theta $(4-8 \mathrm{~Hz})$ and reinforce beta $(13-20 \mathrm{~Hz})$ activity at $\mathrm{Cz}$. The THERAPRAX ${ }^{\circledR}$ EEG Biofeedback system (Neuroconn GmbH, Germany) with a DC-amplifier and a sampling rate of $128 \mathrm{~Hz}$ was used to transmit and analyze the EEG signal. Reference and ground electrodes were attached to right and left mastoids, respectively. Electro-oculogram (EOG) was obtained with two electrodes at external canthi, and two electrodes at infra- and supra-orbital sides. Ocular correction was applied as described in Schlegelmilch et al. [29]. Subsequently, theta/beta index [theta $\left(\mu \mathrm{V}^{2} / \mathrm{Hz}\right)-\operatorname{beta}\left(\mu \mathrm{V}^{2} / \mathrm{Hz}\right) /$ theta $\left.\left(\mu V^{2} / \mathrm{Hz}\right)+\operatorname{beta}\left(\mu \mathrm{V}^{2} / \mathrm{Hz}\right)\right]$ was computed with a

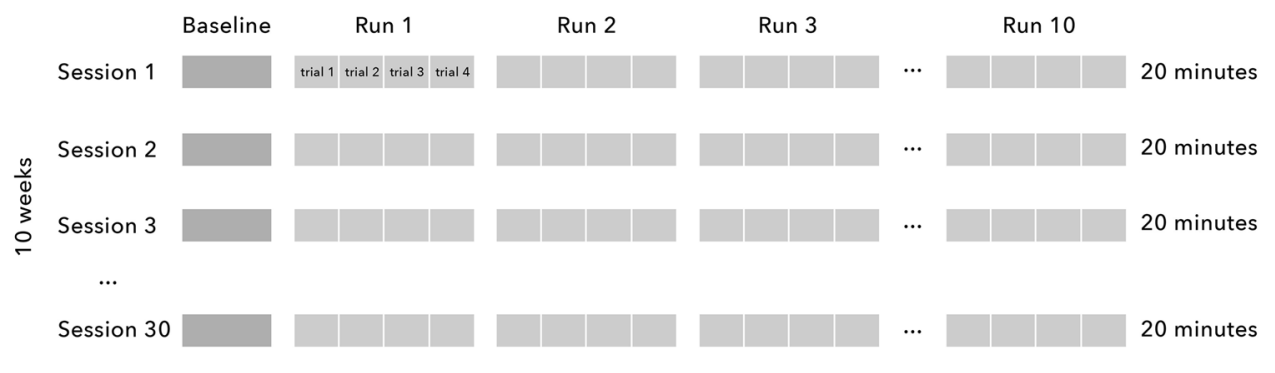

Fig. 1 Schematic representation of the neurofeedback intervention. Approximately 3 sessions of neurofeedback were provided in a week, with 30 sessions taking 10 weeks. Each neurofeedback session started with a 1-min theta/beta baseline recording, which was used for the remaining of the session. A session consisted of 10 runs of 2 min each (20 min effective training). Each run comprised 4 trials of $30 \mathrm{~s}$. Children were instructed to decrease theta/beta compared to the baseline recording during each trial. Children were rewarded with cred- its related to the size of the improvements compared to baseline. The training level increased or decreased based on performance of former runs and ranged between 3 and 52\%. Training level increased after two successive runs with three successful trials each or after one run with four successful trials. Training level decreased after two successive runs with only one successful trial or after one run with no successful trials 
short-time-fourier transformed moving average for direct feedback during runs.

Each run comprised four 30-s trials. The first run of the first session started on a training level with the aim to reduce the theta/beta index with $3 \%$ compared to baseline. The training level increased or decreased based on performance of former runs and ranged between 3 and 52\%. Training level increased after two successive runs with three successful trials each or after one run with four successful trials. Training level decreased after two successive runs with only one successful trial or after one run with no successful trials. From the second session onwards, training level of the first run was set at the second lowest level that was achieved in the former session. Theta/beta index was represented to the participant by simple graphics on a screen. Successful reduction of the theta/beta index as averaged over one trial relative to the baseline was rewarded with the appearance of a sun and granted with credits. The number of earned credits per trial depended on the training level, with more credits for higher levels to reinforce and motivate children to increase performance: 3 and 5\% were rewarded with 1 credit; 10, 12, and $14 \%$ were rewarded with 2 credits; 16,19 , and $23 \%$ were rewarded with 3 credits; 26, 28, and $30 \%$ were rewarded with 4 credits; 33,35 , and $37 \%$ were rewarded with 5 credits; $40,42,45$, and $47 \%$ were rewarded with 6 credits, and 50 and $52 \%$ were rewarded with 7 credits.

Transfer trials without immediate visual feedback were included from session 11 (25\%) and session 21 (50\%) onwards. To further transfer learned behaviors, participants were instructed to retrieve their neurofeedback experiences by watching printed graphics of the training during school and homework. Compliance was verified by questioning the participants whether they used the transfer cards over the intervention period. Transfer cards were used by $84 \%$ of the participants.

\section{Outcome measures}

\section{Training outcomes}

During the neurofeedback sessions the trainer kept track of the training level represented as the percentage decrease in theta/beta index as compared to baseline for each run separately as well as the total number of earned credits per run. Dependent measures included the mean training level over runs within a session (\%), the best run of each session (maximum achieved training level), and the total number of obtained credits per session.

\section{$E E G$}

EEG-recordings were analyzed with Brain Vision Analyzer v2.0 (Brain Products GmbH, Germany). A high-pass filter of $0.5 \mathrm{~Hz}, 12 \mathrm{~dB} /$ octave and a low-pass filter of $30 \mathrm{~Hz}$, $48 \mathrm{~dB} /$ octave were applied. Ocular correction was applied as in Gratton, Coles, and Donchin [30]. Data of the training baselines and runs were segmented in 2-s epochs. Automatic raw data inspection was applied with a maximum allowed voltage step between samples of $50 \mu \mathrm{V} / \mathrm{ms}$, maximum allowed difference of $120 \mu \mathrm{V}$ in each segment, and permitted amplitude range of $-100,100 \mu \mathrm{V}$. Before and after detected artifacts, $200 \mathrm{~ms}$ of data were removed. The lowest permitted activity in intervals was $0.5 \mu \mathrm{V}$ with an interval length of $50 \mathrm{~ms}$. Fast Fourier Transformation (FFT) with a $20 \%$ Hamming window was applied for tapering, and averages over the artifact-free epochs were calculated. Mean power $\left(\mu V^{2}\right)$ was exported to SPSS for the trained frequency bands: theta $4-8 \mathrm{~Hz}$, and beta $13-20 \mathrm{~Hz}$. EEG baseline was available for 93 and $92 \%$ of the training data, respectively.

\section{Behavior}

Raw scores on the scales Inattention and Hyperactivity/ Impulsivity of the Strengths and Weaknesses of ADHD symptoms and Normal behavior scale (SWAN) [31] were used to evaluate the relation between behavioral change and theta- and beta-slopes within and between sessions (see Statistical Analysis).

\section{Procedure}

Prior to the start of the study, approval was obtained from the national medical ethics committee (Ref. no: NL 31641.029.10 CCMO). Children were recruited from 15 child mental health institutions in the West of the Netherlands. Written informed consent was obtained from the parents and children aged 11 years and older. Interventions took place between September 2010 and March 2014. The duration of the intervention period was approximately 10 weeks.

\section{Statistical analysis}

All analyses were performed using SPSS version 21.0 (Corp IBM, released 2012). Values of $p<0.05$ were considered statistically significant. Only significant results are reported. For all outcome measures data were available for at least $92 \%$ of the participants.

Linear mixed models were used to test whether children with ADHD were able to learn to adapt EEG theta and beta activity within and between neurofeedback sessions over the course of a 10 -week, 30 sessions theta/beta neurofeedback intervention. Dependent variables included training outcomes as well as theta and beta power. The linear model included linear fixed effects for session and run, 
Table 2 Outcome measures

\begin{tabular}{|c|c|c|c|c|c|}
\hline & \multicolumn{2}{|c|}{ Available data } & \multirow[t]{2}{*}{ Mean } & \multirow[t]{2}{*}{ SD } & \multirow[t]{2}{*}{ Range } \\
\hline & $N$ & $\%$ & & & \\
\hline Number of sessions followed & 38 & & 28.53 & 2.63 & 19.00 to 30.00 \\
\hline \multicolumn{6}{|l|}{ Training outcomes } \\
\hline Mean training level per session $(\%)$ & 1076 & 99 & 6.64 & 5.61 & 3.00 to 46.20 \\
\hline Max training level per session $(\%)$ & 1076 & 99 & 9.94 & 7.42 & 3.00 to 52.00 \\
\hline Amount of gained credits per session & 1076 & 99 & 28.22 & 32.18 & 0.00 to 220.00 \\
\hline \multicolumn{6}{|l|}{ EEG theta activity } \\
\hline Mean theta baseline $\left(\mu V^{2}\right)$ & 1007 & 93 & 1.71 & 0.40 & 0.30 to 3.78 \\
\hline Mean theta training $\left(\mu V^{2}\right)$ & 10002 & 92 & 1.67 & 0.46 & 0.30 to 4.72 \\
\hline \multicolumn{6}{|l|}{ EEG beta activity } \\
\hline Mean beta baseline $\left(\mu V^{2}\right)$ & 1007 & 93 & 0.67 & 0.16 & 0.21 to 1.78 \\
\hline Mean beta training $\left(\mu V^{2}\right)$ & 10002 & 92 & 0.75 & 0.22 & 0.19 to 2.50 \\
\hline \multicolumn{6}{|c|}{ SWAN parent difference scores: pre- to post-intervention } \\
\hline Inattention & 38 & 100 & -0.32 & 0.66 & -2.22 to 0.78 \\
\hline Hyperactivity/impulsivity & 38 & 100 & -0.29 & 0.68 & -1.67 to 1.44 \\
\hline \multicolumn{6}{|c|}{ SWAN teacher difference scores: pre- to post-intervention } \\
\hline Inattention & 37 & 97 & -0.11 & 0.66 & -1.44 to 1.89 \\
\hline Hyperactivity/impulsivity & 37 & 97 & -0.03 & 0.82 & -2.33 to 1.78 \\
\hline
\end{tabular}

Strengths and Weaknesses of ADHD symptoms and Normal behaviour scale (SWAN)

$S D$ standard deviation

a random intercept over participants, and a random slope for session and run. Exploratory analyses were conducted to assess possible differences in the distribution of transfer trials and feedback trials over the sessions, which were performed with the addition of the fixed factor percentage of transfer trials and the interaction of percentage of transfer trials with session. Additionally, to test whether a parabolic function would increase the fit of the model in addition to linear functions, analyses were performed with the addition of quadratic terms for run and session. Finally, because of possible effects of comorbid disorders on the theta/beta ratio $[32,33]$, sensitivity analyses were performed with exclusion of the children with ADHD and comorbid disorders $(n=7)$.

To explore individual learning curves in the ability to adapt EEG theta or beta activity within and between 30 neurofeedback sessions, separate mixed models were performed for theta and beta power for each participant. The linear model included linear fixed effects for session and run. For each participant, the individual slopes over runs and sessions were used to determine whether there was a significant decrease in theta power and an increase in beta power. Learners were defined as participants able to significantly change theta or beta power in the desired direction.

To explore whether the individual ability to adapt EEG theta or beta activity was related to behavioral changes, linear stepwise regression models were performed with SWAN difference scores (SWAN scores post-intervention minus pre-intervention) as dependent variables. Two sets of independent variables were analyzed: (1) theta slopes over runs and theta slopes over sessions and (2) beta slopes over runs and beta slopes over sessions. SWAN teacher scores were missing for one participant.

\section{Results}

\section{Training outcomes}

Outcome measures for the total group are listed in Table 2. Mean training level increased linearly over the training sessions, $F(1,1040.164)=34.61, p<0.001, b=0.0877$, $95 \% \mathrm{CI}=[0.0585$ to 0.1171$]$, and was accompanied by a linear increase in the maximum obtained training level over the training sessions, $F(1,1041.022)=29.04$, $p<0.001, b=0.1150,95 \% \mathrm{CI}=[0.0731$ to 0.1568$]$. Total number of gained credits also increased over sessions, $F(1,1042.525)=58.08, p<0.001, b=0.7501,95 \%$ $\mathrm{CI}=[0.5570$ to 0.9433$]$.

\section{EEG learning curves}

\section{Theta activity}

Training baseline theta activity did not change over the course of the sessions, $F(1,969.765)=0.412, p=0.521$, $b=-0.0006,95 \% \mathrm{CI}=[-0.0023$ to 0.0012$]$. During 

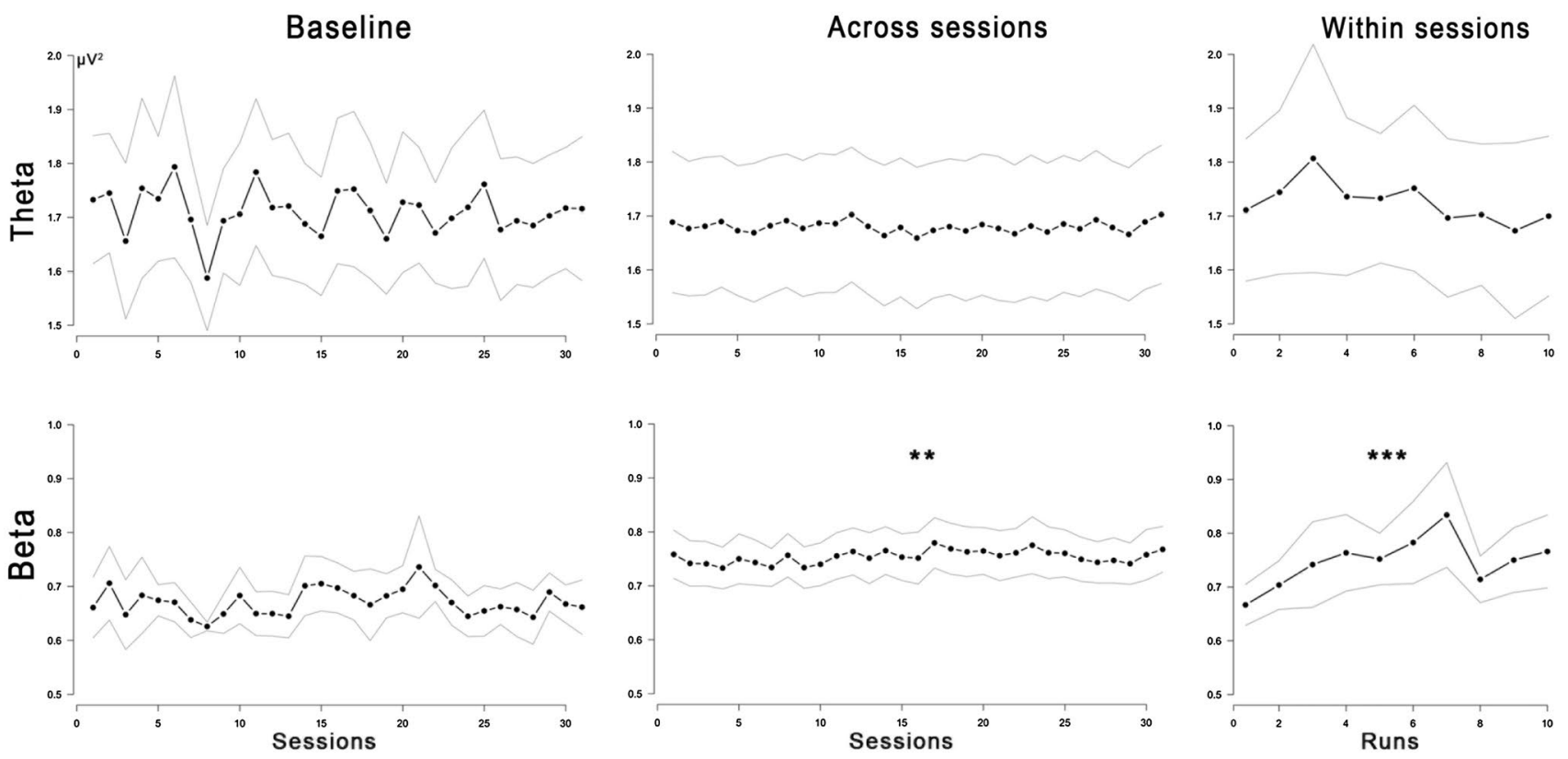

Fig. 2 EEG learning effects during neurofeedback. These graphs show changes in theta and beta power $\left(\mu V^{2}\right)$ during 1-min baseline EEG recordings preceding each neurofeedback session, across ses-

the intervention, theta activity did not change over sessions, $F(1,44.687)=0.322, p=0.573, b=-0.0020$, $95 \% \mathrm{CI}=[-0.0090$ to 0.0050$]$. Furthermore, theta activity did not change over runs within sessions, $F(1$, $1040.445)=1.844, p=0.175, b=-0.0010,95 \%$ $\mathrm{CI}=[-0.0004$ to 0.0023$]$.

\section{Beta activity}

Similar to theta, beta activity during training baseline did not change over the sessions, $F(1,971.583)=1.87$, $p=0.171, b=0.0007,95 \% \mathrm{CI}=[-0.0003$ to 0.0016$]$. In contrast, during the intervention, beta activity showed a linear increase over sessions, $F(1,57.461)=8.60, p=0.005$, $b=0.004095 \% \mathrm{CI}=[0.0013$ to 0.0067$]$. Additionally, beta activity increased linearly over runs within sessions, $F(1,1012.625)=63.51, p<0.001, b=0.005295 \%$ $\mathrm{CI}=[0.0039$ to 0.0065$]$. EEG learning curves are shown in Fig. 2.

\section{Exploratory analyses}

Analyses showed that the percentage of transfer trials during a session did not significantly influence training results in theta and beta bands. Furthermore, quadratic terms did not further improve the linear mixed models. Sensitivity analyses showed that results remained essentially unchanged when analyses were rerun, excluding children with ADHD and comorbid disorders $(n=7)$, indicating that the presence sions (1-30) and within sessions (runs 1-10). The bold lines are means, and the upper and lower lines are $95 \%$ confidence intervals. $* * p<0.01, * * * p<0.001$

of comorbid disorders did not affect the results. Only a nearsignificant effect for baseline beta was found, showing a slight increase over the sessions with exclusion of comorbid disorders, $F(1,796.367)=3.862, p=0.050, b=0.00095$, 95\% CI $=[0.000001$ to 0.001903$]$. Complete statistical details are provided in Supplementary Table 1.

\section{Individual learning curves}

\section{Theta activity}

Over the course of the intervention, $23(61 \%)$ participants showed a negative slope for theta over the sessions of which 15 (39\%) participants showed a significant negative slope and were indicated as theta session learners. However, 7 (18\%) participants showed a significant change over the sessions in the opposite of the desired direction with an increase in theta. Over the runs within sessions, 14 (37\%) of the participants presented a negative slope, although only $4(11 \%)$ of these participants showed a significant negative slope and were indicated as theta run learners. Only one (3\%) participant showed a significant positive theta slope over runs.

\section{Beta activity}

A total of 28 (74\%) participants showed the desired positive beta slope over sessions, of which $20(53 \%)$ participants showed a significant positive slope and were indicated as 
beta session learners. Over the sessions, 3 (8\%) participants showed a significant negative beta slope. Thirty-one (82\%) participants were able to increase beta over runs within sessions and $13(34 \%)$ participants were indicated as beta run learners (showing a significant positive slope). There were no participants that showed a significant negative beta slope over runs.

\section{Behavioral change and EEG slopes}

No significant associations were found between beta or theta individual slopes over sessions or runs and changes in SWAN Inattention and Hyperactivity/impulsivity scales (post- minus pre-intervention) as reported by parents and teachers. Complete statistical details are provided in Supplementary Table 2 .

\section{Discussion}

Theta/beta neurofeedback is aimed at altering brain activity using operant conditioning principles with the goal to improve behavior and neurocognitive functioning in children with ADHD. However, few studies have demonstrated that actual learning takes place during neurofeedback treatment in children with ADHD, which is an essential element for the effectivity of the intervention. The results of the current study provide evidence that children with ADHD learned to decrease the theta/beta index over the sessions in a linear fashion, concordant with the training goals. More detailed analysis of electroencephalogram (EEG) data obtained during the intervention seems to ascribe learning effects primarily to increasing beta power over and within sessions at group level, and at the individual level as well. However, for the theta band, analyses of individual EEG learning curves showed considerable interindividual variation, masking potential effects at group level.

An important general aspect of neurofeedback is a correct translation of learning theory principles to the training design [34]. The current study complies with most of the suggested relevant learning principles, such as proper timing of feedback $(<250 \mathrm{~ms})$, specificity of the trained EEG signal (online EOG correction), shaping (adjusting thresholds and reinforcement magnitude), type of reinforcement (simple graphic), and generalization (successful application of transfer trials and transfer cards), which are probably reflected in the successful training results. It should be noted that this and other comparable studies still have a way to go concerning the specificity of the trained signal. First, knowledge about the functional meaning of theta/beta ratio should be improved. Second, artifacts, such as electromyographic (EMG) activity produced by skeletal muscles, may covertly influence theta/beta ratio $[35,36]$.
In the theta band, we could not demonstrate learning effects at the group level; however, more detailed analysis of individual learning curves showed considerable heterogeneity in training results. While 15 participants significantly learned to reduce theta in accordance with the training goals (39\%), 7 children (18\%) increased theta and 16 children $(43 \%)$ showed no statistically significant training effects over sessions. Only the study by Lubar et al. [22] found comparable results as in our study, showing 12 significant learners $(63 \%)$ over sessions. We identified only few learners in the within-session data, with 4 significant learners in line with the training goals $(11 \%)$, and 1 child showing training effects in the opposite direction. One other study by Bink et al. [25] demonstrated successful suppression of theta within training sessions. An alternative explanation for theta decreases in our study may be the fact that children show a developmental decrease in theta activity over time [37, 38], with a drop in theta activity around the age of nine years [38]. Since the majority was not able to suppress theta within sessions, it is questionable whether the negative individual theta slopes over the intervention indeed result from the neurofeedback intervention or rather originate from developmental changes.

Learning effects were most apparent in the beta frequency band, with linear beta power increases over and within 30 sessions of neurofeedback at group level. At the individual level, more than half of the children were identified as significant beta learners over the sessions, and approximately one-third as significant beta learners over the runs within the sessions. It is difficult to establish whether beta changes represent genuine alterations in brain activity or reflect the reinforcement of artifact data, such as EMG activity [35, 36]. Although the peak frequency of EMG is at relatively high frequencies, the EMG spectrum is very broad and may influence adjacent beta frequencies more than lower frequency bands such as theta. Despite specific instructions during neurofeedback training to prevent excessive muscular tension, it cannot be ruled out that some children used more subtle covert muscular tension to influence the theta/beta ratio. This explanation is further supported by the fact that we could not convincingly demonstrate increases in baseline beta, as recorded before commencing each neurofeedback training, and the lack of chronic beta changes during both rest and task conditions at post-measurement [39]. To reduce potential EMG contamination, higher frequencies may be inhibited, such as in the Lubar protocol [40], or EMG activity could be separately monitored for the major skeletal muscles.

A challenging task is to interpret the various electrophysiological findings of this study. Although we demonstrated learning effects in theta and beta frequency bands, these were not significantly related to symptom improvement in children with ADHD, which may be a necessary prerequisite to demonstrate 
the specificity of the results [21]. Furthermore, the behavioral findings [41] could not confirm the efficacy of theta/beta neurofeedback compared to the control group according to parent and teacher reports. Surprisingly, at post-treatment, children that received theta/beta neurofeedback were characterized by a specific decrease in theta power compared to the control group during a resting eyes open condition, but not during a task condition [27]. This pattern of results may represent chronic effects of the intervention, which do not generalize to a task- and goal-related context, consistent with a lack of event-related potential (ERP) effects during the same task [42]. It may, therefore, be contradictory that in the current study no effects were found for the baseline power spectra measurements, which were performed at the start of each neurofeedback training. This might be explained by the fact that these measurements were of shorter duration, mostly in the afternoon instead of the morning, and in a different context.

A few limitations should be considered when interpreting the findings of the current study. First, although the study contained a random sample of children with ADHD due to the RCT design, a relative large participant sample $(n=38)$, and large number of EEG data points (30 sessions $\times 10$ trials; approximately $10 \mathrm{~h}$ for each participant), the design of the study did not allow to compare neurofeedback with a placebo-controlled condition. Accordingly, this precludes stronger conclusions on the specificity of the findings. Future sham-controlled studies could assess whether theta and beta changes between and within sessions are due to developmental changes and EMG artefacts or not. Second, although training and EEG analyses solely involved the vertex location on the scalp, neurofeedback may alter a more extended cortical region. Future studies may add more electrodes to measure widespread EEG effects during the intervention, while still confining the feedback signal to the vertex. Third, a noticeable qualitative observation of within-session beta effects is a steep decline in beta power at approximately $3 / 4$ of the session (run 8 out of 10) after a linear increase in beta power (run 1 to 7 ; see also Fig. 1). This might indicate that children were not able to remain motivated towards the end of the session or that they could not sustain the energy demands of the training. Future studies may consider these factors, especially considering potential detrimental effects on acquired learning in the first part of the training. Last, cost-effectiveness of neurofeedback could be increased with predictive models of treatment success. Although learning curves were not predictive in the current study, theta power at pre-treatment has been found predictive [27]. It would be worthwhile to search for additive predictive factors.

Overall, the results of the current study show that learning took place during theta/beta neurofeedback in children with ADHD. However, it remains more difficult to interpret these findings, especially since training results were not related to behavioral changes. Future studies are encouraged to obtain electrophysiological training data and to report on the various training components of the intervention. This kind of data can play an important role in developing more effective neurofeedback interventions for ADHD, by isolating trainable components and improving our understanding of the underlying mechanisms of neurofeedback.

Acknowledgements We thank all participating children and families for their contribution, as well as all research interns for their valuable support. Further, we thank the participating centers of child and adolescent psychiatry: ADHD behandelcentrum, Albert Schweitzer ziekenhuis, Alles Kits, GGZ Delfland, GGZ ingeest, Groene Hart ziekenhuis, Groos Mentaal Beter Jong, Kinderpraktijk Zoetermeer, Lucertis, Maasstad ziekenhuis, PuntP, RIAGG Schiedam, and Yulius Academy. This trial was funded by The Netherlands Organization for Health Research and Development (ZonMw): 157003012. ZonMw funded the trial, but had no role in the data analysis, manuscript preparation or decision to publish.

\section{Compliance with ethical standards}

Conflict of interest None of the authors has a conflict of interest in the conduct and reporting of this study.

Open Access This article is distributed under the terms of the Creative Commons Attribution 4.0 International License (http://creativecommons.org/licenses/by/4.0/), which permits unrestricted use, distribution, and reproduction in any medium, provided you give appropriate credit to the original author(s) and the source, provide a link to the Creative Commons license, and indicate if changes were made.

\section{References}

1. American Psychiatric Association (2000) Diagnostic and statistical manual of mental disorders: DSM-IV-TR. American Psychiatric Publishing Inc, Washington, DC

2. American Psychiatric Association (2013) Diagnostic and statitical manual of mental disorders, 5th ed. Washington, DC

3. Faraone SV, Buitelaar J (2010) Comparing the efficacy of stimulants for ADHD in children and adolescents using meta-analysis. Eur Child Adolesc Psychiatry 19:353-364. doi:10.1007/ s00787-009-0054-3

4. Greenhill LL, Gwanson JM, Vitiello B et al (2001) Impairment and deportment responses to different methylphenidate doses in children with ADHD: the MTA titration trial. J Am Acad Child Adolesc Psychiatry 40:180-187. doi:10.1097/00004583-200102000-00012

5. van de Loo-Neus GHH, Rommelse N, Buitelaar JK (2011) To stop or not to stop? How long should medication treatment of attentiondeficit hyperactivity disorder be extended? Eur Neuropsychopharmacol 21:584-599. doi:10.1016/j.euroneuro.2011.03.008

6. Ahmed R, McCaffery KJ, Aslani P (2013) Factors influencing parental decision making about stimulant treatment for attentiondeficit/hyperactivity disorder. J Child Adolesc Psychopharmacol 23:163-178. doi:10.1089/cap.2012.0087

7. Sonuga-Barke EJS, Brandeis D, Cortese S et al (2013) Nonpharmacological interventions for ADHD: systematic review and meta-analyses of randomized controlled trials of dietary 
and psychological treatments. Am J Psychiatry 170:275-289. doi:10.1176/appi.ajp.2012.12070991

8. Cortese S (2012) The neurobiology and genetics of attentiondeficit/hyperactivity disorder (ADHD): what every clinician should know. Eur J Paediatr Neurol 16:422-433. doi:10.1016/j. ejpn.2012.01.009

9. Snyder SM, Hall JR (2006) A meta-analysis of quantitative EEG power associated with attention-deficit hyperactivity disorder. J Clin Neurophysiol 23:440-455. doi:10.1097/01. wnp.0000221363.12503.78

10. Banaschewski T, Brandeis D (2007) Annotation: what electrical brain activity tells us about brain function that other techniques cannot tell us - a child psychiatric perspective. J Child Psychol Psychiatry 48:415-435. doi:10.1111/j.1469-7610.2006.01681.x

11. Loo SK, Barkley RA (2005) Clinical utility of EEG in attention deficit hyperactivity disorder. Appl Neuropsychol 12:64-76. doi:10.1207/s15324826an1202_2

12. Loo SK, Makeig S (2012) Clinical utility of EEG in attentiondeficit/hyperactivity disorder: a research update. Neurotherapeutics 9:569-587. doi:10.1007/s13311-012-0131-z

13. Gevensleben H, Rothenberger A, Moll GH, Heinrich H (2012) Neurofeedback in children with ADHD: validation and challenges. Expert Rev Neurother 12:447-460. doi:10.1586/ern.12.22

14. Lofthouse N, Arnold LE, Hersch S et al (2012) A review of neurofeedback treatment for pediatric ADHD. J Atten Disord 16:351-372. doi:10.1177/1087054711427530

15. Moriyama TS, Polanczyk G, Caye A et al (2012) Evidence-based information on the clinical use of neurofeedback for ADHD. Neurotherapeutics 9:588-598. doi:10.1007/s13311-012-0136-7

16. Gevensleben H, Holl B, Albrecht B et al (2009) Is neurofeedback an efficacious treatment for ADHD? A randomised controlled clinical trial. J Child Psychol Psychiatry 50:780-789. doi:10.1111/j.1469-7610.2008.02033.x

17. Steiner NJ, Frenette EC, Rene KM et al (2014) In-school neurofeedback training for ADHD: sustained improvements from a randomized control trial. Pediatrics 133:483-492. doi:10.1542/ peds.2013-2059

18. Arnold LE, Lofthouse N, Hersch S et al (2013) EEG neurofeedback for ADHD: double-blind sham-controlled randomized pilot feasibility trial. J Atten Disord 17:410-419. doi:10.1177/1087054712446173

19. Perreau-Linck E, Lessard N, Lévesque J, Beauregard M (2010) Effects of neurofeedback training on inhibitory capacities in ADHD children: a single-blind, randomized, placebo-controlled study. J Neurother 14:229-242. doi:10.1080/10874208.2010.501 514

20. van Dongen-Boomsma M, Vollebregt MA, Slaats-Willemse D, Buitelaar JK (2013) A randomized placebo-controlled trial of electroencephalographic (EEG) neurofeedback in children with attention-deficit/hyperactivity disorder. J Clin Psychiatry 74:821-827. doi:10.4088/JCP.12m08321

21. Zuberer A, Brandeis D, Drechsler R (2015) Are treatment effects of neurofeedback training in children with ADHD related to the successful regulation of brain activity? A review on the learning of regulation of brain activity and a contribution to the discussion on specificity. Front Hum Neurosci 9:135. doi:10.3389/ fnhum.2015.00135

22. Lubar JF, Swartwood MO, Swartwood JN, O’Donnell PH (1995) Evaluation of the effectiveness of EEG neurofeedback training for ADHD in a clinical setting as measured by changes in T.O.V.A. scores, behavioral ratings, and WISC-R performance. Biofeedback Self Regul 20:83-99

23. Monastra VJ, Monastra DM, George S (2002) The effects of stimulant therapy, EEG biofeedback, and parenting style on the primary symptoms of attention-deficit/hyperactivity disorder. Appl Psychophysiol Biofeedback 27:231-249
24. Leins U, Goth G, Hinterberger T et al (2007) Neurofeedback for children with ADHD: a comparison of SCP and theta/beta protocols. Appl Psychophysiol Biofeedback 32:73-88. doi:10.1007/ s10484-007-9031-0

25. Bink M, van Nieuwenhuizen C, Popma A et al (2014) Behavioral effects of neurofeedback in adolescents with ADHD: a randomized controlled trial. Eur Child Adolesc Psychiatry. doi:10.1007/s00787-014-0655-3

26. Gevensleben H, Holl B, Albrecht B et al (2009) Distinct EEG effects related to neurofeedback training in children with ADHD: a randomized controlled trial. Int J Psychophysiol 74:149-157. doi:10.1016/j.ijpsycho.2009.08.005

27. Janssen TWP, Bink M, Geladé K et al (2016) A randomized controlled trial into the effects of neurofeedback, methylphenidate, and physical activity on EEG power spectra in children with ADHD. J Child Psychol Psychiatry 57:633-644. doi:10.1111/ jcpp. 12517

28. Ogrim G, Hestad KA (2013) Effects of neurofeedback versus stimulant medication in attention-deficit/hyperactivity disorder: a randomized pilot study. J Child Adolesc Psychopharmacol 23:448-457. doi:10.1089/cap.2012.0090

29. Schlegelmilch F, Markert S, Berkes S, Schellhorn K (2004) Online ocular artifact removal for dc-EEG-signals: estimation of dc-level. Biomed Tech 2:340-341

30. Gratton G, Coles M, Donchin E (1983) A new method for offline removal of ocular artifact. Electroencephalogr Clin Neurophysiol 55:468-484

31. Swanson JM, Schuck S, Mann M et al (2001) Categorical and dimensional definitions and evaluations of symptoms of ADHD: The SNAP and the SWAN rating scales

32. Loo SK, Cho A, Hale TS et al (2013) Characterization of the theta to beta ratio in ADHD: identifying potential sources of heterogeneity. J Atten Disord 17:384-392. doi: $10.1177 / 1087054712468050$

33. Snyder SM, Rugino T a., Hornig M, Stein MA (2015) Integration of an EEG biomarker with a clinician's ADHD evaluation. Brain Behav 5:n/a-n/a. doi: 10.1002/brb3.330

34. Sherlin LH, Arns M, Lubar J et al (2011) Neurofeedback and basic learning theory: implications for research and practice. J Neurother 15:292-304. doi:10.1080/10874208.2011.623089

35. Goncharova I, McFarland D, Vaughan T, Wolpaw J (2003) EMG contamination of EEG: spectral and topographical characteristics. Clin Neurophysiol 114:1580-1593. doi:10.1016/ S1388-2457(03)00093-2

36. McMenamin BW, Shackman AJ, Greischar LL, Davidson RJ (2011) Electromyogenic artifacts and electroencephalographic inferences revisited. Neuroimage 54:4-9. doi:10.1016/j. neuroimage.2010.07.057

37. Gasser T, Verleger R, Bächer P, Sroka L (1988) Development of the EEG of school-age children and adolescents. I. Analysis of band power. Electroencephalogr Clin Neurophysiol 69:91-99. doi:10.1016/0013-4694(88)90204-0

38. Somsen RJM, van't Klooster BJ, van der Molen MW et al (1997) Growth spurts in brain maturation during middle childhood as indexed by EEG power spectra. Biol Psychol 44:187-209. doi: 10.1016/S0301-0511(96)05218-0

39. Janssen TWP, Bink M, Gelad K et al (2016) A randomized controlled trial into the effects of neurofeedback, methylphenidate, and physical activity on EEG power spectra in children with ADHD. J Child Psychol Psychiatry Allied Discip 57:633-644. doi:10.1111/jepp.12517

40. Lubar JO, Lubar JF (1984) Electroencephalographic biofeedback of SMR and beta for treatment of attention deficit disorders in a clinical setting. Biofeedback Self Regul 9:1-23

41. Geladé K, Janssen TWP, Bink M, et al (2016) Behavioral effects of neurofeedback compared to stimulants and physical activity in 
attention-deficit/hyperactivity disorder: a randomized controlled trial. J Clin Psychiatry

42. Janssen TWP, Bink M, Geladé K, et al (2016) A randomized controlled trial investigating the effects of neurofeedback, methylphenidate, and physical activity on event-related potentials in children with attention-deficit/hyperactivity disorder. J Child Adolesc Psychopharmacol 26:cap.2015.0144. doi: 10.1089/cap.2015.0144 